\title{
Simulated Annealing Parallel Genetic Algorithm Based on Building Blocks Migration
}

\author{
Zhiyong Li and Xilu Zhu \\ School of computer and communication, Hunan University, China, 410082

\begin{abstract}
Through analyzing schema theorem and building blocks theory, propose parallel genetic algorithms based on building blocks migration. Relying on convergence condition, receive building blocks from other populations. Using simulated annealing method prevent the density of good schema to increase greatly which will result in premature convergence. Theory analysis and experimental results show that the method not only reduce ineffective migration and decrease communication costs, but also lower the possibility of occurring premature and assure the capability of global convergence.
\end{abstract}

Keywords: parallel genetic algorithm, schema theorem, building blocks theory, simulated annealing, migration strategy.

\section{Introduction}

In recent years, many specialists have been researching and made significant contributions in PGA[1]. However, How to improve algorithm performance[2] and the extensibility of PGA are still barriers blocking PAG advancing[3].

In this paper, we introduce a new migration strategy which base on building blocks theory and integrate Boltzmann survivor mechanism to reduce communication cost and enhance coarse-grained PGA searching efficiency.

\section{Building Block Operator}

To guarantee to find global solution, the population should contain at least one same-defining-position-non-overlapping set, which can completely cover the whole solution area.

Definition 1. If population exists a building blocks[4] set $\left\{H_{1}, H_{2} \cdots H_{n}\right\}$, and satisfy $H_{a} \cap H_{b}=\varnothing$, where $\forall a, b \in\{1,2 \cdots k\}$. The intersection is null if and only if no identical value in the same locus. Therefore the set mentioned above is non-overlapping BBs set.

Set threshold $\theta_{0}, S(1)=\sum_{j=1}^{n} x_{i j}$ where $x_{i j}=1 . P(1)=S(1) / n$.If $P(1)>\theta_{0}$, the value 1 is accepted to make up BBs, otherwise $1-\mathrm{P}(1)>\theta_{0}$, the value 0 is accepted to make up BBs, else accept $*$. 


\section{An Outline of Simulated Annealing Parallel Genetic Algorithm Based on Building Blocks Migration}

STEP 1. Initialize population $S^{L}=\{0,1\}^{L}$ randomly, and calculate population fitness. STEP 2. If existing migrations do follows; else return the global solution.

STEP 3. If $i_{\text {loot }}$, when children fitness lower than parents, we consider premature convergence in GA process, and do STEP 4.else continue GA process.

STEP 4. When GA proceeds slowly, it means that subpopulation converge to a local optimum, we extract building block $H_{l}$, and select BBs which nearly or exactly match the principle of non-overlapping.

STEP 5. Use Metropolis strategy[5] to accept building blocks which migrate from other population. The equation is described below by:

$$
p_{\text {accept }}=\left\{\begin{array}{cl}
\min \left(1, \exp \left(\frac{f_{\text {migrator }}-\bar{f}}{T}\right)\right)>\operatorname{random}[0,1) & f_{\text {migrator }} \leq \bar{f} \\
1 & f_{\text {migrator }}>\bar{f}
\end{array}\right.
$$

As the temperature of annealing fall, the probability of accepting bad solution diminishes. The Metropolis avoids GA to trap in local optimum.

\section{Communication Costs}

In coarse grained PGA, overall cost V[6] estimated casually is described below as:

$$
V=\left[\frac{g}{M I}-2\right] \times \sum_{j=1}^{m} r_{i} s_{i} b
$$

However the overall cost of BBsAPGA described below by equation:

$$
V^{\prime}=\left[\frac{g}{C}\right] \times m b
$$

$\mathrm{C}$ denote premature interval. Obviously, $C=n M I$.

$$
\Delta V=V-V^{\prime}=\left[\frac{g}{M I}-2\right] \times \sum_{i=1}^{g} r_{i} s_{i} b-\left[\frac{g}{C}\right] \times m b
$$

Therefore, BBsAPGA effectively lower communication cost, decrease ineffective number of migration.

\section{Experimental Results}

To investigate the parallel efficiency of PGA and genetic operators, we conduct experiments on a cluster. All computations are performed on HP P4(3G),512 memory. 


$$
f=100-\left[\left(4-2.1 x^{2}+\frac{x^{4}}{3}\right) x^{2}+x y+\left(-4+4 y^{2}\right) y^{2}\right] x, y \in[-2.048,2.048]
$$

Where the function has 6 local optimums, two of them are global optimum

$$
f=\cos (5 x) \cos (5 y) e^{-0.001\left(x^{2}+y^{2}\right)} \quad x, y \in[-2.048,2.048]
$$

Where the function has many local optimum, $f(0,0)=1$ is the global optimum

Table 1. Experimental results

\begin{tabular}{|c|c|c|c|c|}
\hline experiment & $\begin{array}{c}\text { PGA } \\
\text { migration }\end{array}$ & $\begin{array}{c}\text { BBsAPGA } \\
\text { migration }\end{array}$ & $\begin{array}{c}\text { PGA } \\
\text { convergence rate }\end{array}$ & $\begin{array}{c}\text { BBsAPGA } \\
\text { convergence rate }\end{array}$ \\
\hline 1 & 670 & 31 & 55 & 33 \\
\hline 2 & 1170 & 33 & 95 & 73 \\
\hline
\end{tabular}

The result of experiment manifest BBsAPGA effectively lower communication cost, decrease ineffective number of migration. Meanwhile this algorithm overcome premature convergence phenomena, accelerate evolution, enhance global searching.

\section{Conclusions}

Analyze schema theorem and building blocks theory deduced by schema theorem, introduce building block operator. Integrating Boltzmann survivor mechanism propose a simulated annealing parallel genetic algorithm based on building blocks. The BBsAPGA reduce ineffective migration, decrease cost of communication. Evidently it lower premature probability and ensure GA global convergence.

\section{References}

1. Muhlenbein, H.,Schomisch, M. and Born,J.: The parallel genetic algorithm as function optimizer. Proceedings of the Fourth International Conference on Genetic Algorithms (1991) 619-632.

2. Nowostawski M., Poli R.: Parallel genetic algorithm taxonomy. Proceedings of the Third International conference on knowledge-based intelligent information engineering systems (KES'99) (1999) 88-92.

3. Cantu-Paz E.: A survey of parallel genetic algorithms. Calculateurs Paralleles, Vol. 10. Hermes Paris (1998) 141-171.

4. Lee A.: The schema theorem and Price's theorem. Foundations of Genetic Algorithms . Morgan Kaufmann San Francisco (1995) 23-49

5. Emile Aarts, Jan Korst.: Simulated annealing and Boltzmann machines : a stochastic approach to combinatorial optimization and neural computing. John Wiley and Sons New York (1989)

6. Baowen $\mathrm{Xu}, \mathrm{Yu}$ Guan, Zhenqiang Chen.: Parallel Genetic Algorithms with Schema Migration. Proceedings of 26th International Computer Software and Applications Conference (COMPSAC 2002) (2002) 879-884 